\title{
Ductile to brittle transition in dynamic fracture of brittle bulk metallic glass
}

\author{
G. Wang, ${ }^{1}$ Y. N. Han, ${ }^{1}$ X. H. Xu, ${ }^{2}$ F. J. Ke, ${ }^{2}$ B. S. Han, ${ }^{1}$ and W. H. Wang ${ }^{1, a)}$ \\ ${ }_{1}^{1}$ Institute of Physics, Chinese Academy of Sciences, Beijing 100080, People's Republic of China \\ ${ }^{2}$ Department of Applied Physics, Beihang University, Beijing 100083, People's Republic of China \\ and State Key Laboratory of Nonlinear Mechanics, Institute of Mechanics, Chinese Academy of Sciences, \\ Beijing 100080, People's Republic of China
}

(Received 28 November 2007; accepted 26 February 2008; published online 7 May 2008)

\begin{abstract}
We report an unusual transition from a locally ductile to a pure brittle fracture in the dynamic fracture of brittle $\mathrm{Mg}_{65} \mathrm{Cu}_{20} \mathrm{Gd}_{10}$ bulk metallic glass. The fractographic evolution from a dimple structure to a periodic corrugation pattern and then to the mirror zone along the crack propagation direction during the dynamic fracture process is discussed within the framework of the meniscus instability of the fracture process zone. This work might provide an important clue in understanding of the energy dissipation mechanism for dynamic crack propagation in brittle glassy materials.

(C) 2008 American Institute of Physics. [DOI: 10.1063/1.2912491]
\end{abstract}

\section{INTRODUCTION}

The catastrophic brittle fracture of glassy materials is one of the most poorly understood fundamental phenomena in materials science. ${ }^{1-6}$ Kelly et $a l^{2}{ }^{2}$ concluded that metallic solids plastically deform at crack tips, whereas brittle amorphous materials, such as glassy polymers and oxide glasses, generally support perfectly brittle cracks; the fundamental mechanism of brittle fracture is one of sequential bond rupture. This view was further strengthened by the theory of Rice $e t$ al. $^{2}$ and experimental observations that claim that the fracture of glasses at a crack tip occurs via ductile separation at the nanoscale, ${ }^{4}$ while Guin and Wiederhorn ${ }^{5}$ found that at a crack tip, glasses exhibit a pure brittle fracture. Xi et al. ${ }^{6}$ found a dimplelike structure on the fracture surface of brittle bulk metallic glasses (BMGs) and concluded that local softening is the main mechanism controlling the fracture behavior of brittle BMGs. More recently, a periodic corrugation pattern was observed on the fracture surface of various brittle BMGs. ${ }^{7-9}$ However, it remains to be experimentally seen whether these fracture characteristics are generic in a brittle fracture. The fractographic evolution along the crack propagation during dynamic fracture of brittle BMGs has not been investigated, which might be an important clue to elucidate the energy dissipation during dynamic crack propagation. ${ }^{1}$

In this paper, we use brittle $\mathrm{Mg}_{65} \mathrm{Cu}_{25} \mathrm{Gd}_{10}$ BMG [with a fracture toughness $K_{C}$ value of 1-2 $\mathrm{MPa} \mathrm{m}^{1 / 2}$ (Ref. 6)] as a model material to investigate the fractographic evolution with dynamic crack extension. We find an unusual fractographic evolution from a nanoscale dimplelike structure to nanoscale periodic corrugations and then to a flat mirror zone along the crack propagation direction, which suggests that a ductile to a pure brittle transition occurs during the dynamic fracture. The results indicate that the fracture surface roughness evolution is different from previous experimental and theoretical results and might be useful for in-depth understanding of the fracture mechanism of brittle glasses.

\footnotetext{
a) Author to whom correspondence should be addressed. Electronic mail: whw@ aphy.iphy.ac.cn.
}

\section{EXPERIMENTAL PROCEDURES}

The fracture surfaces of a cylindrical BMG $(3 \mathrm{~mm}$ in diameter and $16 \mathrm{~mm}$ in length) with single-edge notches were prepared by three-point bending test on an Instron machine with crosshead moving velocities $V$ of $0.1,1$, and 10 $\mathrm{mm} / \mathrm{min}$ at ambient conditions. The notch radius prepared by diamond saw is about $250 \mu \mathrm{m}$. The larger notch radius can significantly increase the toughness of the glass. ${ }^{6}$ At each crosshead moving velocity, the bending test was repeated three to five times to check consistency. The newly created fracture surfaces were examined by using a Philips XL30 scanning electron microscope (SEM) with a resolution of 1.5 $\mathrm{nm}$ and a Digital NanoScope IIIa D-3000 atomic force microscope (AFM).

\section{RESULTS AND DISCUSSION}

The fractographic evolution tendency and the average size of dimplelike structure and spacing of periodic corrugation along the crack propagation distance (see Fig. 2) are almost the same for all the specimens. The applied stress state in the crack tip during crack propagation generated by three-point bending test is described elsewhere. ${ }^{10}$ The average crack propagation speed of the metallic glass under the crosshead moving speed of $1 \mathrm{~mm} / \mathrm{min}$ is estimated to be about $1000 \mathrm{~m} / \mathrm{s}^{11}$ The fracture surface profile for the displacement velocity of $V=1 \mathrm{~mm} / \mathrm{min}$ as observed by SEM is shown in Fig. 1. From the notch point to the edge, the fracture surface shows four zones with different fractographic features: dimple structure zone [Fig. 1(b)], transition zone consisting of a mixture of dimple pattern and periodic corrugation pattern [Fig. 1(c)], periodic corrugation pattern zone [Figs. 1(d) and 1(e)], and mirror zone [Fig. 1(f)].

To further quantitatively characterize the dependence of the fractographic evolution on the crack propagation distance, we capture a series of fracture surface SEM images at different spots along the crack propagation direction, such as along the dot arrow in Fig. 1(a), in all of the three specimens with different displacement velocities. The interval distance between two spots in the dimplelike structure zone is ap- 

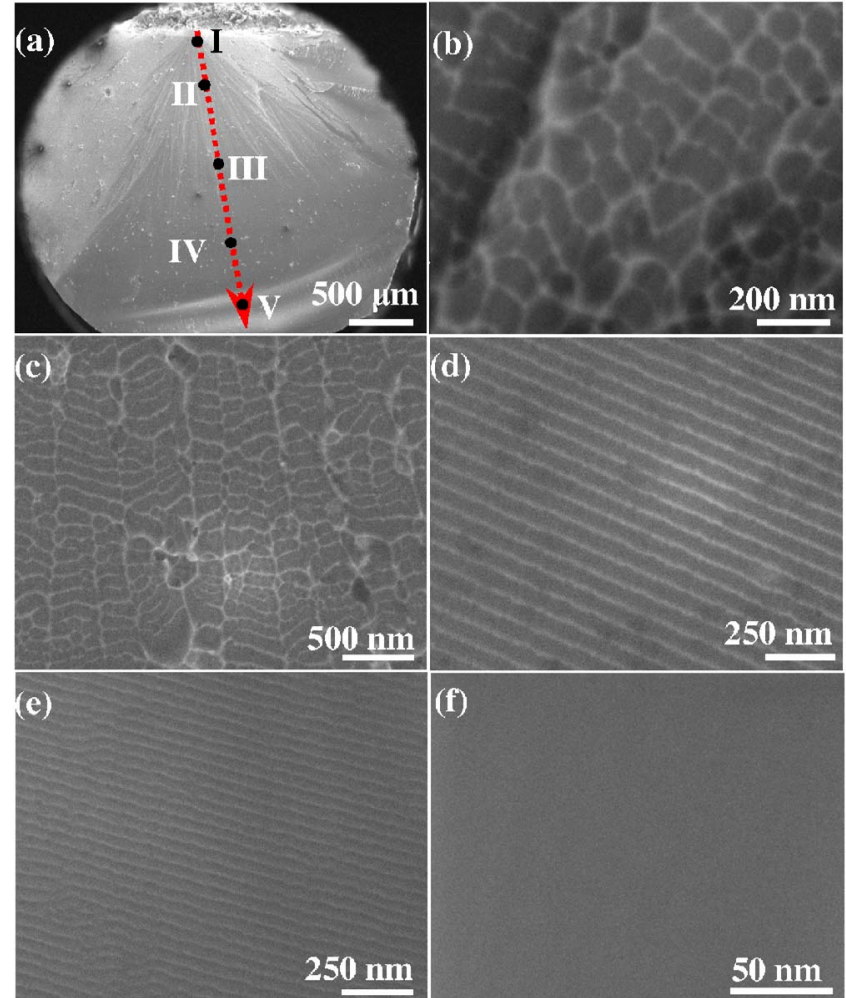

(f)

FIG. 1. (Color online) Fracture surface profile of the BMG bent at the crosshead moving velocity of $1 \mathrm{~mm} / \mathrm{min}$. (a) Overview of the fracture surface (the red arrow indicates the crack propagation direction). (b) Dimplelike structure on the notch point [point I in (a)]. (c) Image for the transition zone on point II in (a). (d) Periodic corrugation pattern on point III in (a). (e) Periodic corrugation pattern on point IV in (a). (e) Mirror zone on point $\mathrm{V}$ in (a).

proximately 10 or $30 \mu \mathrm{m}$. In the other zones, i.e., the transition zone and the corrugation zone, it is approximately 100 or $200 \mu \mathrm{m}$. Through directly measuring the size of the dimple and the corrugation from these SEM images at different spots, the dependences of the dimple size and the corrugation spacing on the crack propagation distance bent with three different velocities can be plotted, as shown in Fig. 2. With increasing $V$, the dimple size gets smaller, while the transition zone becomes bigger. In the transition zone, the dimple size or the corrugation spacing increases with in-

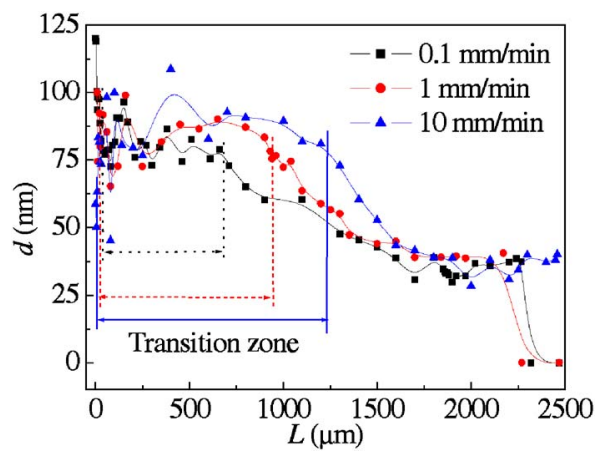

FIG. 2. (Color online) Size of the dimplelike structure and spacing of periodic corrugation $d$ as a function of crack propagation distance $L$ for three specimens bent with different $V$ values. The solid spots represent the size of the dimplelike structure or the spacing of corrugation that was directly measured from the SEM images.

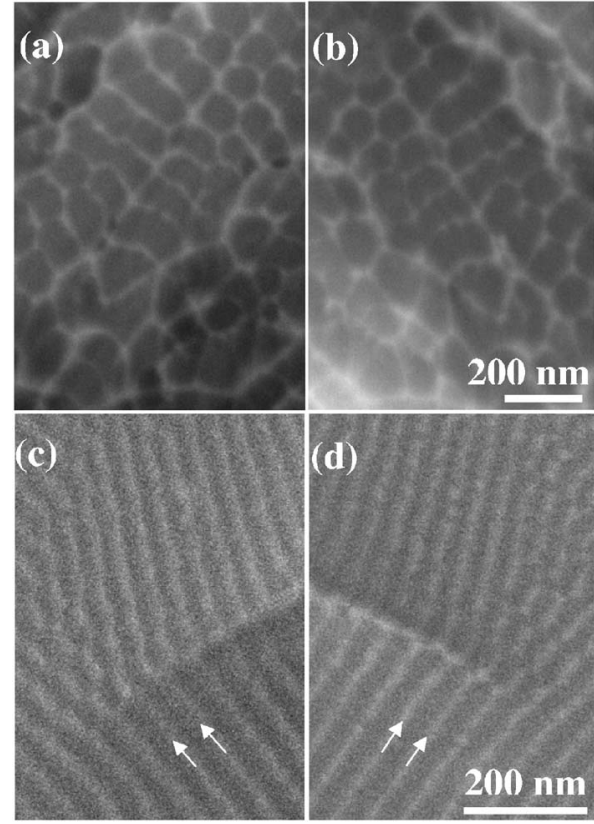

FIG. 3. Fractographic analysis on the matching fracture surfaces of the bent BMG. [(a) and (b)] Matching dimplelike structure zones on opposite fracture surfaces. [(c) and (d)] Matching periodic corrugation pattern corrugation zones on opposite fracture surface.

creasing $V$. Once the crack passes the transition zone, the spacings of the corrugation bent with different velocities reduce to $\sim 38 \mathrm{~nm}$. With further crack propagation, the periodic corrugation spacing eventually reduces to zero and the mirror zone appears. The dimplelike structure zone always appears near the notch and is independent of the test velocity because the crack propagation process is a quasistatic state at this stage, and the formation of the dimple zone depends on the fracture process zone size in the front of the crack tip. ${ }^{6}$ The formation of periodic corrugation is due to a dynamic crack propagation that needs to surpass a critical distance $l_{C}$ and be beyond a critical crack propagation speed $V_{C}{ }^{9,12}$ The fractographic evolution of the BMG bent with $0.1 \mathrm{~mm} / \mathrm{min}$ is the same as that observed in Fig. 1, while when the BMG was bent with $10 \mathrm{~mm} / \mathrm{min}$, no mirror zone is observed.

The SEM observations shown in Figs. 3(a)-3(d) indicate that the dimplelike structure and the periodic corrugations exhibit a peak-to-peak separation at the crack tip, which has also been confirmed by AFM [shown in Figs. 4(a) and 4(b) $].{ }^{11}$ This indicates that the fracture at the crack tip processes a ductile deformation. ${ }^{6}$ The morphology of the mirror zone observed by high resolution SEM is featureless [Fig. 1(f)]. AFM observations confirm that the roughness of the mirror zone is less than $0.5 \mathrm{~nm}$, and no dimple or periodic corrugation can be found [Figs. 4(a) and 4(b)]. This means that the local softening mechanism does not dominate the fracture behavior at the crack tip in the mirror zone, where a pure brittle fracture similar to that in brittle silica glasses occurs. ${ }^{5}$ The fractographic evolution from a dimplelike structure to a periodic corrugation pattern and then to the mirror zone along the crack propagation direction indicates a ductile to a brittle transition during the dynamic fracture process of brittle BMG.

The temperature rise $\Delta T$ within the fracture process zone 

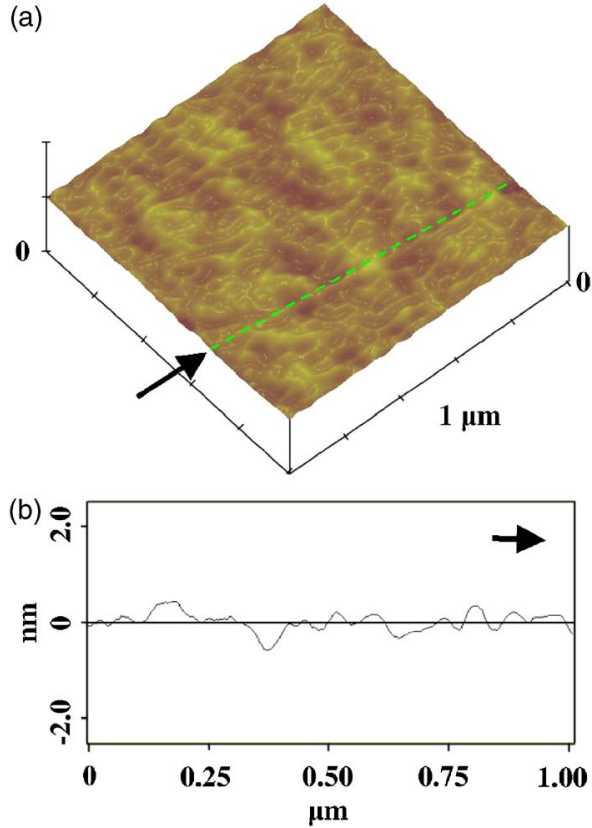

FIG. 4. (Color online) (a) Three-dimensional image of the mirror zone. (b) Section shape of roughness along a dashed line in (a).

is estimated by a function mentioned by Rice et al.: ${ }^{12} \Delta T$ $=\left[1.414\left(1-\nu^{2}\right) K_{c} \sigma_{Y} \sqrt{V}\right] /(E \sqrt{\rho c k})$, where $\rho$ is the mass density $\left(3.79 \times 10^{6} \mathrm{~g} / \mathrm{m}^{3}\right), c$ is the thermal capacity, $\nu$ is Poisson's ratio (0.313), $E$ is the elastic modulus (50.6 GPa), $\sigma_{Y}$ is the yield stress (672 MPa), and $k$ is the thermal conductivity. The thermal capacity roughly equals to $1 \mathrm{~J} / \mathrm{g} \mathrm{K}$ (Ref. 13) and thermal conductivity is approximately $5 \mathrm{~W} / \mathrm{m} \mathrm{K}$ and is weakly composition dependent. ${ }^{14}$ The maximum crack speed $V$ should not exceed the Rayleigh wave speed, $V_{R}$ $\approx 0.9225 c_{s}$, where $c_{s}$ is the shear wave speed $(2254 \mathrm{~m} / \mathrm{s}) .{ }^{15}$ The maximum temperature rise for $\mathrm{Mg}$-based $\mathrm{BMG}$ is estimated to be $355 \mathrm{~K}$, which is in the supercooled liquid region, where the glassy phase behaves as a viscoelastic body. ${ }^{16} \mathrm{Ac}$ tually, the local heating and excess free volume aggregation driven by deformation are widely observed in various BMGs. ${ }^{17-19}$ Therefore, the crack propagation process could be simplified as a finger advancing into a viscous matter behaving as a viscoelastic body in the fracture process zone (with the size of $\Delta$ ) following the Saffman-Taylor model, as shown in Fig. 5. ${ }^{20,21}$ Once the dynamic crack propagation

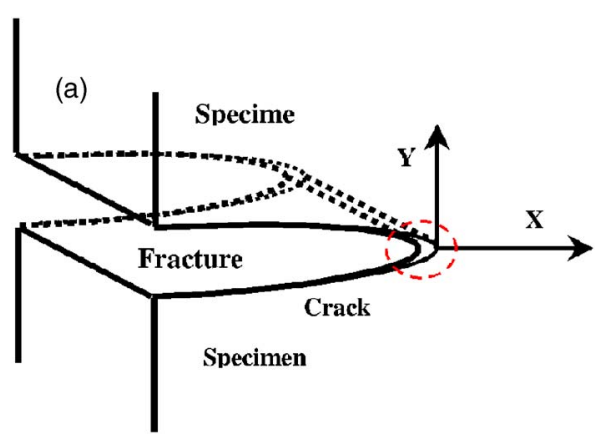

FIG. 5. (Color online) Sketch illustrating the fracture process zone in the front of the crack tip (crack propagating along $x$ axis). Three-dimensional sketch of the crack propagation. The fracture process zone is located in the crack tip, which is enclosed by a circle.

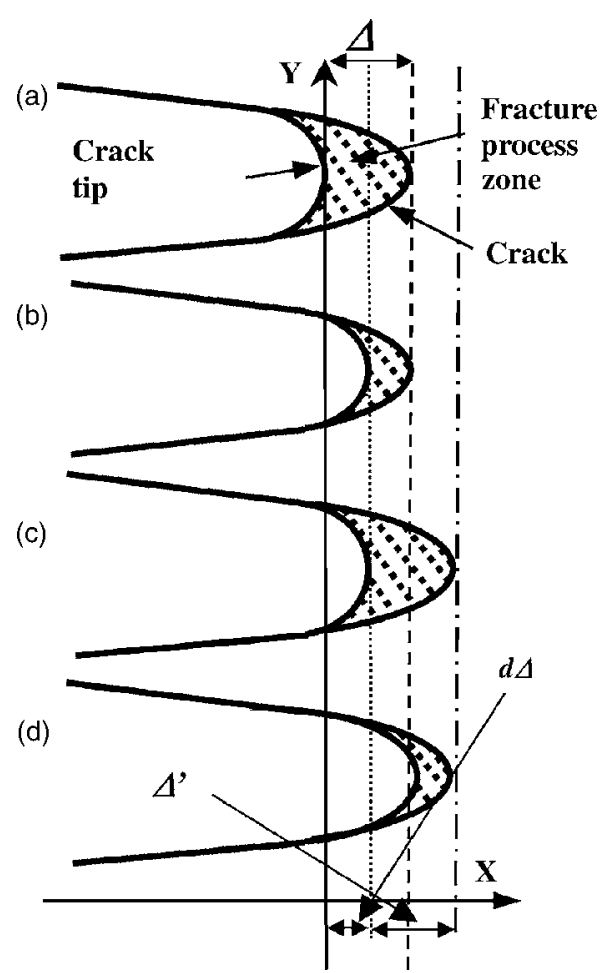

FIG. 6. The illustration of the fracture process zone evolution is simplified into two dimension sketches in (a)-(d). (a) Original state of the crack propagation. The $\Delta$ represents the size of the fracture process zone in the front of the crack tip. (b) The crack tip penetrates into the fracture process zone and an internal stress is built up. The depth of the penetration is $d \Delta$. (c) The fracture process zone advances into the specimen driven by the internal stress and the size of the fracture process zone recovers to $\Delta^{\prime}$. (d) Relaxing process of the viscoelastic medium is prohibited. The fast running crack tip penetrates into the specimen body.

process is set up, the glassy phase in the fracture process zone behaves as a viscoelastic body, which exhibits three different characteristics depending on the crack propagation speed. $^{22}$ At a lower speed (near the notch), the crack tip slowly advances into the fracture process zone and the viscous matter in this zone has enough time to rearrange, and the fracture process zone can keep its original size of $\Delta$ [Fig. 6(a)]. The cavities will nucleate and coalesce to form the dimple structure in the front of crack tip, as shown in Fig. 2(b). ${ }^{23,24}$ At a high speed, the viscous matter exhibiting a viscoelastic behavior has no time to rearrange and will be compressed. The viscoelastic medium acts as an elasticlike deformation and the internal stress will be built up in the compressed viscoelastic medium. The size of the fracture process zone is reduced to $\Delta-d \Delta$ [Fig. 6(b)]. Once the crack tip advances a critical distance or the internal stress reaches a critical value, the crack tip advancing will be stopped and the internal stress will drive the fracture process zone advancing. As a result, the fracture process zone recovers to the original size $\Delta^{\prime}$ [Fig. 6(c)]. As the internal stress is relaxed, the crack tip will again penetrate into the fracture process zone in the next cycle. In a dynamic process, the compressible and relaxing processes of the viscoelastic medium strongly depend on the strain rate, i.e., the crack speed. If the crack speed is further increased, the viscoelastic medium does not have enough time to fully relax and the compressed zone cannot recover to its original size, i.e., $\Delta^{\prime}<\Delta$, and the formed in- 
ternal stress cannot be fully relaxed either, and the $d \Delta$ is then reduced in next cycle. When the fracture process zone $(\Delta$ $-d \Delta)$ is less than the wavelength of the meniscus instability, the dimplelike structure will be suppressed and the periodic corrugation pattern appears. With $\Delta^{\prime}$ reducing with increasing crack speed, the spacing of the periodic corrugation decreases, as shown in Figs. 1(d) and 1(e), because the spacing is correlated with the fracture process zone, $\Delta^{\prime} .{ }^{11}$ Once the crack speed reaches the critical value, the relaxation is completely suppressed and the viscoelastic medium dominates the crack tip advancing. In this case, the cycle processes in the crack tip front cannot be processed; instead, the crack propagation is replaced by a fast running crack tip penetrating into the specimen body and the fracture process zone should be compressed into a very short range $\left(\Delta^{\prime} \rightarrow 0\right)$, and the mirror zone appears, as shown in Fig. 6(d).

The model can also explain the critical length for formation of the periodic corrugation (or the presentation of the dimple zone). The critical length can be predicted by the equation of motion for a single-edge crack in a semi-infinite body as ${ }^{25} l_{C}=(\Gamma E) /\left[3.939 \sigma^{2}\left(1-V_{C} / V_{R}\right)\right]$, where $\Gamma$ is the material's fracture energy, $V_{R}$ is the Rayleigh wave speed, $E$ is the elastic modulus, and $\sigma$ is the fracture stress. Since $E$, $\Gamma$, and the critical crack speed are constant values, $l_{c}$ depends on the fracture stress. Experimental results show that the fracture stress increases from 85.2 to $135.4 \mathrm{MPa}$ with increasing $V$. As such, $l_{c}$ decreases with increased $\sigma$, as shown in Fig. 2.

The fractographic evolution in most brittle glassy materials exhibits a characteristic pattern sequence known as an initially smooth and mirrorlike fracture surface followed by a misty and then a highly rough hackled region. ${ }^{26}$ The violent oscillation, beginning once the mean speed passes a welldefined critical value, generates a rough pattern and is well correlated with spatial structure on the newly created crack surface. $^{27,28}$ The microcracking behavior and the crack front wave or shear wave affecting on the crack front were proposed to elucidate the roughness of dynamic fracture surface. ${ }^{29}$ However, the present results show a different morphologic sequence, i.e., dimplelike structure zone, transition zone, periodic corrugation zone, and mirror zone, with increasing crack speed. The results indicate that the fracture behavior of brittle BMG in the crack tip transforms from ductile (softening mode) to pure brittle fracture (in mirror zone) depending on the dynamic fracture process. Our model can qualitatively explain the unusual fractographic evolution along the crack propagation direction and predict the change tendency of the spacing of the periodic corrugation.

Some materials, such as refractory metals and steel, also exhibit both types of behavior with a brittle to a ductile transition at a characteristic temperature. ${ }^{30-33}$ While the experimental evidence on the controlling factors of the transition remains inconclusive. The local fracture behavior transition from ductile to pure brittle we observed may highlight commonality and contrasts the brittle to the ductile transition phenomenon and sheds new light on the mechanism of the transition. The ductile mechanism has been widely observed for a large variety of metallic alloys, including most BMGs. ${ }^{6}$ It was concluded that metallic solids plastically deform at crack tips, but that covalent or ionic glasses will generally support perfectly brittle cracks. ${ }^{2,34}$ Against the conventional belief, we report experimental evidence that metallic glasses could break like oxide glasses in some cases. The similarity between the fracture models even in some special cases of the two different types of metallic and covalent glasses could provide an important clue in understanding of the basic energy dissipation mechanism for fracture in brittle glassy materials.

\section{CONCLUSIONS}

We observe unusual fractographic evolution along the crack propagation direction indicating a local fracture behavior of brittle BMG during transformation from locally ductile to pure brittle. This transformation is due to the propagation speed dependent dynamic behavior of the viscoelastic matter in the crack tip front where the local softening is stimulated in the fracture process zone. The dynamic behavior is set up when the crack surpasses a critical distance and dominates the size of the fracture process zone. Once the crack speed reaches a high value, the relaxation of the viscoelastic matter is suppressed and then the periodic corrugation formation in the fracture process zone also is frozen. The mirror zone on the fracture surface or the pure brittle fracture occurs. Considering that many brittle glasses such as oxide glasses exhibit mirror, mist, and hackle, it remains to be experimentally seen whether such an unusual fractographic evolution we have observed is generic in other brittle metallic glasses.

We thank Professor Y.L. Bai for a critical reading of the manuscript, M.X. Pan and D.Q. Zhao for discussions and the support of the NSFC (Grant Nos. 50621061 and 50731008) and MOST 973 (Grant No. 2007CB613904).

${ }^{1}$ J. Fineberg and M. Marder, Phys. Rep. 313, 1 (1999).

${ }^{2}$ A. Kelly, W. R. Tyson, and A. H. Cottrell, Philos. Mag. 15, 576 (1967); J. R. Rice and R. Thomson, ibid. 29, 73 (1974).

${ }^{3}$ J. J. Lewandowski, W. H. Wang, and A. L. Greer, Philos. Mag. Lett. 85, 77 (2005); J. J. Lewandowski, Mater. Trans., JIM 42, 633 (2001).

${ }^{4}$ F. Célarié, S. Prades, D. Bonamy, L. Ferrero, E. Bouchaud, C. Guillot, and C. Marliére, Phys. Rev. Lett. 90, 075504 (2003).

${ }^{5}$ J.-P. Guin and S. M. Wiederhorn, Phys. Rev. Lett. 92, 215502 (2004).

${ }^{6}$ X. K. Xi, D. Q. Zhao, M. X. Pan, W. H. Wang, Y. Wu, and J. J. Lewandowski, Phys. Rev. Lett. 94, 125510 (2005); W. H. Wang, J. Appl. Phys. 99, 093506 (2006); W. H. Wang, C. Dong, and C. H. Shek, Mater. Sci. Eng., R. 44, 45 (2004).

${ }^{7}$ G. Wang, Y. T. Wang, Y. H. Liu, M. X. Pan, D. Q. Zhao, and W. H. Wang, Appl. Phys. Lett. 89, 121909 (2006); G. Wang, D. Q. Zhao, H. Y. Bai, M. X. Pan, A. L. Xia, B. S. Han, X. K. Xi, Y. Wu, and W. H. Wang, Phys. Rev. Lett. 98, 235501 (2007).

${ }^{8}$ J. Shen, W. Z. Liang, and J. F. Sun, Appl. Phys. Lett. 89, 121908 (2006).

${ }^{9}$ X. K. Xi, D. Q. Zhao, M. X. Pan, W. H. Wang, Y. Wu, and J. J. Lewandowski, Appl. Phys. Lett. 89, 181911 (2006).

${ }^{10}$ H. Tada, P. C. Paris, and G. R. Irwin, The Stress Analysis of Cracks Handbook (Del Research Corporation, Hellertown, PA, 1973), pp. 2.162.17 .

${ }^{11} \mathrm{G}$. Wang, X. H. Xu, and W. H. Wang (unpublished).

${ }^{12} \mathrm{~J}$. R. Rice and N. Levy, in The Physics of Strength and Plasticity, edited by A. S. Argon (MIT, Cambridge, MA, 1969), p. 286.

${ }^{13}$ C. T. Liu, L. Heatherly, D. S. Easton, C. A. Carmichael, J. H. Schneibel, C. H. Chen, J. L. Wright, M. H. Yoo, J. A. Horton, and A. Inoue, Metall. Mater. Trans. A 29, 1811 (1998).

${ }^{14}$ L. Li, T. Lin, C. Dong, and J. J. Lin, Phys. Rev. B 74, 172201 (2006)

${ }^{15}$ K. Ravi-Chandar and B. Yang, J. Mech. Phys. Solids 45, 535 (1997).

${ }^{16}$ J. Lu, G. Ravichandran, and W. L. Johnson, Acta Mater. 51, 3429 (2003). 
${ }^{17}$ F. Spaepen, Acta Metall. 25, 407 (1977).

${ }^{18}$ B. Yang, M. L. Morrison, P. K. Liaw, R. A. Buchanan, G. Y. Wang, C. T. Liu, and M. Denda, Appl. Phys. Lett. 86, 141904 (2005).

${ }^{19}$ J. J. Lewandowski and A. L. Greer, Nat. Mater. 5, 15 (2006); Y. Zhang, N. A. Stelmashenko, Z. H. Barber, W. H. Wang, J. J. Lewandowski, and A. L. Greer, J. Mater. Res. 22, 419 (2007).

${ }^{20}$ A. S. Argon and M. Salama, Mater. Sci. Eng. 23, 219 (1976).

${ }^{21}$ D. A. Kurtze and D. C. Hong, Phys. Rev. Lett. 71, 847 (1993).

${ }^{22}$ H. L. Roche, J. F. Fernandez, H. Octavio, A. G. Loeser, and C. Lobb, Phys. Rev. A 44, R6185 (1991); E. Lamaire, P. Levitz, G. Daccord, and H. V. Damme, Phys. Rev. Lett. 67, 2009 (1991).

${ }^{23}$ M. Kanninen, W. Adler, W. Rosenfield, and R. Jaffee, Inelastic Behavior of Solids (McGraw-Hill, New York, 1969), p. 641.

${ }^{24}$ W. M. Garrison and N. R. Moody, J. Phys. Chem. Solids 48, 1035 (1987).

${ }^{25}$ A. Livne, O. Ben-David, and J. Fineberg, arXiv.:cond-mat/0609273 (unpublished).
${ }^{26}$ C. R. Kurkjian, Strength of Inorganic Glass (Plenum, New York, 1985), p. 221.

${ }^{27}$ A. K. Green and P. L. Pratt, Eng. Fract. Mech. 6, 71 (1974).

${ }^{28}$ J. Fineberg, Phys. Rev. Lett. 67, 457 (1991).

${ }^{29}$ D. Bonamy and K. Ravi-Chandar, Phys. Rev. Lett. 91, 235502 (2003); E. Sharon, G. Cohen, and J. Fineberg, Nature (London) 410, 68 (2001).

${ }^{30}$ J. D. Rigney and J. J. Lewandowski, Metall. Mater. Trans. A 27A, 3292 (1996).

${ }^{31}$ A. Samant and J. J. Lewandowski, Metall. Mater. Trans. A 28A, 2297 (1997).

${ }^{32}$ M. G. Mendiratta, R. Goetz, D. M. Dimiduk, and J. J. Lewandowski, Metall. Mater. Trans. A 26A, 1767 (1995).

${ }^{33}$ P. Gumbsch, J. Riedle, A. Hartmaier, and H. F. Fischmeister, Science 282, 1293 (1998).

${ }^{34}$ B. Lawn, Fracture of Brittle Solids, 2nd ed. (Cambridge University Press, Cambridge, 1993), p. 143. 